\title{
On the regularity of weak solutions to Burgers' equation with finite entropy production
}

\author{
Xavier Lamy ${ }^{*} \quad$ Felix Otto ${ }^{\dagger}$
}

\begin{abstract}
Bounded weak solutions of Burgers' equation $\partial_{t} u+\partial_{x}\left(u^{2} / 2\right)=0$ that are not entropy solutions need in general not be $B V$. Nevertheless it is known that solutions with finite entropy productions have a $B V$-like structure: a rectifiable jump set of dimension one can be identified, outside which $u$ has vanishing mean oscillation at all points. But it is not known whether all points outside this jump set are Lebesgue points, as they would be for $B V$ solutions. In the present article we show that the set of non-Lebesgue points of $u$ has Hausdorff dimension at most one. In contrast with the aforementioned structure result, we need only one particular entropy production to be a finite Radon measure, namely $\mu=$ $\partial_{t}\left(u^{2} / 2\right)+\partial_{x}\left(u^{3} / 3\right)$. We prove Hölder regularity at points where $\mu$ has finite $(1+\alpha)$-dimensional upper density for some $\alpha>0$. The proof is inspired by a result of De Lellis, Westdickenberg and the second author : if $\mu_{+}$has vanishing 1dimensional upper density, then $u$ is an entropy solution. We obtain a quantitative version of this statement: if $\mu_{+}$is small then $u$ is close in $L^{1}$ to an entropy solution.
\end{abstract}

\section{Introduction}

It is well-known that weak solutions of Burgers' equation

$$
\partial_{t} u+\partial_{x} \frac{u^{2}}{2}=0
$$

(and more generally scalar conservation laws) are not uniquely determined by initial data, and this is the reason why the notion of entropy solution was introduced [23]. Entropy solutions are characterized by their nonpositive entropy production : for any convex entropy $\eta: \mathbb{R} \rightarrow \mathbb{R}$ and associated entropy flux $q(u)=\int^{u} v \eta^{\prime}(v) d v$, the corresponding entropy production $\mu_{\eta}$ satisfies

$$
\mu_{\eta}=\partial_{t} \eta(u)+\partial_{x} q(u) \leq 0 .
$$

\footnotetext{
*Institut de Mathématiques de Toulouse, Université Paul Sabatier, Toulouse, France. Part of this work was conducted while XL was a postdoctoral researcher at the Max Planck Institute for Mathematics in the Sciences, Leipzig, Germany. Email: xlamy@math.univ-toulouse.fr

†Max Planck Institute for Mathematics in the Sciences, Leipzig, Germany. Email: otto@mis.mpg.de
} 
This constraint ensures well-posedness of the Cauchy problem for (1) with $L^{\infty}$ initial data. Entropy solutions can be equivalently characterized by Oleinik's estimate $\partial_{x} u \leq 1 / t[29]$, and in particular they are locally in $B V$.

Although entropy solutions are the physically relevant solutions, general weak solutions sometimes need to be considered. For instance in [33, 27, 5] large deviation principles for stochastic approximation of entropy solutions are related to variational principles for energy functionals of the form

$$
F_{\varepsilon}(u)=\left.\int\left|\frac{1}{\varepsilon}\right| \partial_{x}\right|^{-1}\left(\partial_{t} u+\partial_{x} \frac{u^{2}}{2}\right)-\left.\varepsilon \partial_{x} u\right|^{2} .
$$

The $\Gamma$-limit of such functional is defined for weak solutions of (1) that need not be entropy solutions, but have finite entropy production:

$$
\mu_{\eta}=\partial_{t} \eta(u)+\partial_{x} q(u) \quad \text { is a locally finite Radon measure, }
$$

for any $\eta \in C^{2}(\mathbb{R})$ and associated flux $q$. An important feature of such solutions is that they enjoy a kinetic formulation (see e.g. [11]), namely there exists $m(t, x, v)$ a locally finite Radon measure such that

$$
\partial_{t} \chi+v \partial_{x} \chi=\partial_{v} m, \quad \chi(t, x, v)=\mathbb{1}_{0<v \leq u(t, x)}-\mathbb{1}_{u(t, x) \leq v<0} .
$$

The measure $m$ encodes the entropy production through the formula

$$
\left\langle\mu_{\eta}, \varphi\right\rangle=\int \eta^{\prime \prime}(v) \varphi(t, x) m(d t, d x, d v)
$$

For entropy solutions it is nonpositive and the kinetic formulation was introduced in $[26]$.

Another motivation for studying general weak solutions of (1) comes from a formal analogy with solutions of the eikonal equation

$$
|\nabla \varphi|=1
$$

that need not be viscosity solutions. Such solutions arise for instance in the problem of $\Gamma$-convergence of the Aviles-Giga functional

$$
E_{\varepsilon}(\varphi)=\frac{\varepsilon}{2} \int\left|\nabla^{2} \varphi\right|^{2}+\frac{1}{2 \varepsilon} \int\left(|\nabla \varphi|^{2}-1\right)^{2} .
$$

They can be endowed with a relevant concept of entropy production $[22,2,15,18]$ and a kinetic formulation $[20,19]$. The $\Gamma$-limit of $E_{\varepsilon}$ is conjectured to be the total entropy production, but a proof of the upper bound is still missing because not enough is known about the regularity of solutions with finite entropy production (see $[6,30]$ when $\nabla \varphi \in B V$ ). The analogy between (1) and (3) has already proven fruitful. For instance, techniques developed in [10] to understand the fine structure of solutions of (3) were adapted in [11] to the context of scalar conservation laws. See also $[21,9]$ for other regularity properties shared by both equations. 
Unlike entropy solutions, weak solutions of (1) with finite entropy production (2) may not be in $B V$. They are in $B_{3, \infty}^{1 / 3}$ [17], but this is the best regularity one could hope for [14]. However it is shown in [24, 11] (related results can be found e.g. in $[4,31])$ that they do enjoy a $B V$-like structure, namely: there exists an $\mathcal{H}^{1}$-rectifiable set $\mathcal{J} \subset \Omega$ such that $u$ has strong one-sided traces on $\mathcal{J}$, and vanishing mean oscillation at all points outside $\mathcal{J}$. Moreover the entropy production restricted to the "jump set" $\mathcal{J}$ can be computed with the chain rule: if $\nu$ denotes a normal vector along $\mathcal{J}$ and $u^{ \pm}$the corresponding one-sided traces of $u$, then

$$
\mu_{\eta}\left\lfloor\mathcal{J}=\left[\left(\eta\left(u^{+}\right)-\eta\left(u^{-}\right)\right) \nu_{t}+\left(q\left(u^{+}\right)-q\left(u^{-}\right)\right) \nu_{x}\right] \mathcal{H}^{1}\lfloor\mathcal{J} .\right.
$$

The similarity with the structure of $B V$ solutions is not perfect, and the two following questions are left open:

- Is $\mu_{\eta}$ supported on $\mathcal{J}$ ?

- Is every point outside $\mathcal{J}$ a Lebesgue point of $u$ ?

In the present article we investigate the second question. Note that for entropy solutions of a large class of one-dimensional scalar conservation laws the corresponding questions have been answered positively [13].

The quadratic entropy $\eta(u)=u^{2} / 2$ plays a special role in our analysis. In fact our methods are strongly inspired by [12] where the importance of that particular entropy is shed light upon. We consider bounded weak solutions $u(t, x)$ of (1) in a domain $\Omega \subset \mathbb{R}_{t} \times \mathbb{R}_{x}$ and denote simply by $\mu$ the corresponding entropy production

$$
\mu=\partial_{t} \frac{u^{2}}{2}+\partial_{x} \frac{u^{3}}{3} \in \mathcal{M}(\Omega) .
$$

In [11] the singular set $\mathcal{J}$ is defined as the set of points with positive upper $\mathcal{H}^{1}$ density with respect to the measure $\nu \in \mathcal{M}(\Omega)$ given by

$$
\nu(A)=|m|(A \times \mathbb{R})=\sup _{\left|\eta^{\prime \prime}\right| \leq 1}\left|\mu_{\eta}\right|(A) \quad \text { for } A \subset \Omega .
$$

In other words, denoting by $Q_{r}(z)$ the square of size $r$ centered at $z$, i.e.

$$
Q_{r}(z)=(t-r, t+r) \times(x-r, x+r) \text { if } z=(t, x),
$$

the "regular points" of [11] are those belonging to

$$
\mathcal{J}^{c}=\left\{z \in \Omega: \lim _{r \rightarrow 0} r^{-1} \nu\left(Q_{r}(z)\right)=0\right\} \subset\left\{z \in \Omega: \lim _{r \rightarrow 0} r^{-1}|\mu|\left(Q_{r}(z)\right)=0\right\} .
$$

The last inclusion follows from $|\mu| \leq \nu$, and it is not clear weather it is strict or not.

Remark 1. For $B V$ solutions of (1) the measures $\mu, m$ and $\nu$ can be computed explicitly using the chain rule (see e.g. [5, Remark 2.7]) and one can check that $\nu=|\mu|$ so that the inclusion is not strict. 
In the present paper we need a geometric rate of decay for $r^{-1}|\mu|\left(Q_{r}(z)\right)$ - but no bound on $r^{-1} \nu\left(Q_{r}(z)\right)$ - to conclude that $z$ is a Lebesgue point: our regular points are given by

$$
\widetilde{\mathcal{J}}^{c}=\left\{z \in \Omega: r^{-1}|\mu|\left(Q_{r}(z)\right)=\mathcal{O}\left(r^{\alpha}\right) \text { for some } \alpha>0\right\} .
$$

In order to quantify the regularity we obtain outside of $\widetilde{\mathcal{J}}$ we define, for any $\alpha, K>0$,

$$
\Omega_{\alpha, K}=\left\{z \in \Omega:|\mu|\left(Q_{r}(z)\right) \leq K r^{1+\alpha}, \forall r \in(0, d(z) \wedge 1)\right\},
$$

where $d(z)=\operatorname{dist}_{\infty}\left(z, \Omega^{c}\right)$ denotes the distance of $z$ to the boundary of $\Omega$ with respect to the $\ell^{\infty}$ norm. In particular

$$
\tilde{\mathcal{J}}=\bigcap_{\alpha, K>0}\left(\Omega_{\alpha, K}\right)^{c}
$$

has Hausdorff dimension at most one since $\mathcal{H}^{1+\alpha}\left(\left(\Omega_{\alpha, K}\right)^{c}\right) \lesssim K^{-1}|\mu|(\Omega)$, as follows from a covering argument (see e.g. [3, Theorem 2.56]). In $\Omega_{\alpha, K}$ the function $u$ is Hölder continuous:

Theorem 2. For any bounded weak solution u of (1) with finite entropy production (4), any $\alpha, K>0$ and $z \in \Omega_{\alpha, K}$ it holds

$$
f_{Q_{r}(z)}\left|u-f_{Q_{r}(z)} u\right| \leq C r^{\frac{\alpha}{256}}, \quad \text { for all } r \in(0, d(z)),
$$

where $C>0$ depends on $\|u\|_{L^{\infty}}, K, \alpha$ and $d(z)$.

Remark 3. Such Campanato decay implies local Hölder continuity in $\Omega_{\alpha, K}$ in the classical sense: for any $d_{0}>0$ and $z_{1}, z_{2} \in \Omega_{\alpha, K} \cap\left\{d \geq d_{0}\right\}$ it holds

$$
\left|u\left(z_{1}\right)-u\left(z_{2}\right)\right| \leq C\left|z_{1}-z_{2}\right|^{\frac{\alpha}{256}}
$$

where $C>0$ depends on $\|u\|_{L^{\infty}}, K, \alpha$ and $d_{0}$. Also, note that the explicit dependence on $K, \alpha$ and $d_{0}$ can be infered from the proof in $\S 3$.

Corollary 4. The set of non-Lebesgue points of any bounded weak solution $u$ of (1) with finite entropy production (4) has Hausdorff dimension at most one.

The proof of Theorem 2 relies on the following principle : if the positive part of the entropy production (4) is small, then $u$ should be close to an entropy solution. This principle is already present in [12] where it is shown that if $\mu_{+}$has vanishing upper $\mathcal{H}^{1}$-density, then $u$ must be an entropy solution. Here we obtain, using methods inspired by [12], a quantitative version of this result: (a small power of) the total mass of $\mu_{+}$controls the $L^{1}$-distance of $u$ to entropy solutions. This is the content of the next result, where we write $Q_{r}$ for $Q_{r}(0,0)$. 
Theorem 5. Let $u \in L^{\infty}\left(Q_{1}\right)$ be a weak solution of (1) with finite entropy production (4). Then there exists an entropy solution $\zeta \in L^{\infty}\left(Q_{1}\right)$ of (1) such that

$$
\int_{Q_{3 / 4}}|u-\zeta| \leq C\left(1+|\mu|\left(Q_{1}\right)\right)^{\frac{1}{5}} \mu_{+}\left(Q_{1}\right)^{\frac{1}{64}}
$$

where $C>0$ only depends on $\|u\|_{L^{\infty}}$.

To prove Theorem 5, the main step is to estimate the distance to entropy solutions in a rather weak sense, as explained below. This weak estimate can then be strengthened to an $L^{1}$ estimate by appropriately quantifying the compactness enforced by (4).

Remark 6. If the measure $\nu$ defined in (5), that encodes all entropy productions, is finite, then we have a $B_{3, \infty}^{1 / 3}$ estimate [17] so that the compactness is easily quantified. Such an assumption would simplify the proof of Theorem 5 and improve the dependence on $\mu_{+}\left(Q_{1}\right)$ : we would obtain

$$
\int_{Q_{3 / 4}}|u-\zeta| \leq C \mu_{+}\left(Q_{1}\right)^{\frac{1}{24}}
$$

for some constant $C>0$ depending on $\|u\|_{L^{\infty}}$ and $\nu\left(Q_{1}\right)$. Modifying the definition of the sets $\Omega_{\alpha, K}$ accordingly (i.e. incorporating the constraint $r^{-1} \nu\left(Q_{r}(z)\right) \leq K$ ) this would also yield in Theorem 2 an exponent $\alpha / 96$ instead of $\alpha / 256$.

As in [12] we make use of the correspondence between Burgers' equation and the related Hamilton-Jacobi equation

$$
\partial_{t} h+\frac{1}{2}\left(\partial_{x} h\right)^{2}=0
$$

obtained from observing that the vector field $\left(-u^{2} / 2, u\right)$ is curl-free and therefore can be written as a gradient field $\left(\partial_{t} h, \partial_{x} h\right)$. The aforementioned weak estimate consists in estimating the $L^{\infty}$-distance of $h$ to viscosity solutions of (7), which correspond to entropy solutions of (1) [12]. This is the very heart of our argument and we achieve this in $\S 2$ by turning the following loose statement into a rigorous one: if the positive part of the entropy production is small, then $h$ is "not far" from being a viscosity supersolution of

$$
\partial_{t} h+\frac{1}{2}\left(\partial_{x} h\right)^{2} \geq-\delta, \quad \text { for "small" } \delta .
$$

If $h$ really was a viscosity supersolution of such modified (7), then the comparison principle [8] would allow to estimate its $L^{\infty}$-distance to viscosity solutions. We prove instead a weak version of the maximum principle (Lemma 8) where we need to assume some additional regularity on the subsolution to compare $h$ with, but this turns out to be sufficient for our purposes.

The plan of the article is as follows. In $\S 2$ we derive the estimates for $h$, and in $\S 3$ we prove Theorems 5 and 2 . 


\section{Estimates for the Hamilton-Jacobi equa- tion}

We denote by $Q$ the unit square

$$
Q:=(0,1)_{t} \times(0,1)_{x},
$$

and consider Lipschitz functions $h$ in $\bar{Q}$ that solve (7) almost everywhere. In particular this ensures $[25, \S 11.1]$ that $h$ restricted to the parabolic boundary

$$
\partial_{0} Q:=\{0\}_{t} \times(0,1)_{x} \cup(0,1)_{t} \times\{0,1\}_{x},
$$

is compatible with the existence of a viscosity solution $\bar{h}$ satisfying $\bar{h}=h$ on $\partial_{0} Q$. Moreover, such viscosity solution satisfies $\bar{h} \geq h$ and

$$
\left|\partial_{x} \bar{h}\right| \leq\left\|\partial_{x} h\right\|_{L^{\infty}(Q)} .
$$

For a proof of (8) see Appendix A. The main result of this section is the following estimate for $\|h-\bar{h}\|_{\infty}$.

Proposition 7. Let $t_{1} \in[0,1)$ and $L \geq 1$ be fixed. There exists a constant $C>0$ such that, for any function $h$ with $\operatorname{Lip}(h) \leq L$ solving

$$
\partial_{t} h+\frac{1}{2}\left(\partial_{x} h\right)^{2}=0 \quad \text { a.e. in } Q,
$$

if $u=\partial_{x} h$ is such that $\mu=\partial_{t}\left(u^{2} / 2\right)+\partial_{x}\left(u^{3} / 3\right)$ is a Radon measure in $Q$, then it holds

$$
\sup _{Q \cap\left\{t \leq t_{1}\right\}}|h-\bar{h}| \leq C\left\|\mu_{+}\right\|^{1 / 8},
$$

where $\bar{h}$ is the viscosity solution of

$$
\partial_{t} \bar{h}+\frac{1}{2}\left(\partial_{x} \bar{h}\right)^{2}=0, \quad \bar{h}=h \text { on } \partial_{0} Q .
$$

As explained in the introduction, the proof of Proposition 7 is about showing that if $\mu_{+}$is small, then $h$ is "not far" from being a viscosity supersolution of (7) with small negative right-hand side. Such property is interesting because super- and subsolutions in the viscosity sense enjoy a comparison principle. In fact instead of proving a supersolution property, we directly prove a comparison principle. The main difference with the comparison principle for viscosity solutions is that we have to assume some additional regularity on the subsolution we are comparing $h$ with, namely semiconvexity. We say that a function $\zeta$ is $(1 / r)$-semiconvex if for all points $z, z^{\prime}$ it holds

$$
\zeta\left(\theta z+(1-\theta) z^{\prime}\right)-\theta \zeta(z)-(1-\theta) \zeta\left(z^{\prime}\right) \leq \frac{1}{r}, \quad 0 \leq \theta \leq 1 .
$$

This is equivalent to $\zeta(z)+|z|^{2} /(2 r)$ being convex, and allows to prove the following maximum principle. 
Lemma 8. For all $L \geq 0$ there exists a constant $C>0$ such that for any convex open set $U \subset \mathbb{R}_{t} \times \mathbb{R}_{x}$ the following holds true.

- Let a function $h$ with $\operatorname{Lip}(h) \leq L$ solve

$$
\partial_{t} h+\frac{1}{2}\left(\partial_{x} h\right)^{2}=0 \quad \text { a.e. in } U,
$$

and, denoting $u=\partial_{x} h$ and $\mu=\partial_{t}\left(u^{2} / 2\right)+\partial_{x}\left(u^{3} / 3\right)$, assume that $\mu$ is a Radon measure in $U$.

- For some $\delta, r \in(0,1]$, let a function $\zeta$ with $\operatorname{Lip}(\zeta) \leq L$ be a viscosity subsolution of

$$
\partial_{t} \zeta+\frac{1}{2}\left(\partial_{x} \zeta\right)^{2} \leq-\delta \quad \text { in } U
$$

with the additional regularity assumption that $\zeta$ be $(1 / r)$-semiconvex.

- If the positive entropy production is small enough in the sense that

$$
C\left\|\mu_{+}\right\| \leq \delta^{7} r
$$

then for any $\left(t_{0}, x_{0}\right) \in U$ and $\rho>0$ such that $B_{\rho}\left(t_{0}, x_{0}\right) \subset U$ and $\rho \geq \delta^{2} r$, the function $(h-\zeta)$ restricted to $B_{\rho}\left(t_{0}, x_{0}\right)$ cannot attain its minimum at $\left(t_{0}, x_{0}\right)$.

With Lemma 8 at hand, Proposition 7 will follow by regularizing $\bar{h}$ (using sup-convolution) and appropriately balancing the scale of regularization with the smallness of $\mu_{+}$and the smallness of the negative right-hand side modification of (7).

Proof of Lemma 8. We assume that $L=1$, the general case entailing no additional difficulty. Suppose that (10) holds for some constant $C>0$, and that $(h-\zeta)$ restricted to some ball $B_{\rho}\left(t_{0}, x_{0}\right) \subset U$ with $\rho \geq \delta^{2} r$, attains its minimum at $\left(t_{0}, x_{0}\right)$. We are going to obtain a contradiction if the constant $C$ is large enough. We use coordinates in which $\left(t_{0}, x_{0}\right)=(0,0)$, and assume without loss of generality that $h(0,0)=\zeta(0,0)=0$.

Step 1. There exists an affine function $\zeta_{a}$ with $\operatorname{Lip}\left(\zeta_{a}\right) \leq 1$ and such that

$$
\begin{aligned}
& \partial_{t} \zeta_{a}+\frac{1}{2}\left(\partial_{x} \zeta_{a}\right)^{2} \leq-\delta, \\
& \zeta_{a}(0,0)=\zeta(0,0), \quad \zeta(t, x) \geq \zeta_{a}(t, x)-\frac{1}{2 r}\left(t^{2}+x^{2}\right) .
\end{aligned}
$$

At any point $(t, x)$ where $\zeta$ admits a Taylor expansion

$$
\zeta(t+s, x+y)=\zeta(t, x)+\partial_{t} \zeta(t, x) s+\partial_{x} \zeta(t, x) y+\mathcal{O}\left(s^{2}+y^{2}\right),
$$

the function $(s, y) \mapsto \zeta(t+s, x+y)-\varphi(s, y)$ with

$$
\varphi(s, y)=\partial_{t} \zeta(t, x) s+\partial_{x} \zeta(t, x) y+M\left(s^{2}+y^{2}\right),
$$


admits, provided $M$ is large enough, a local maximum at $(0,0)$. Since $\zeta$ is a viscosity subsolution, by definition of the latter property (see e.g. [12, Definition 2.2]) we deduce that at any such point $(t, x)$ it holds

$$
\partial_{t} \zeta(t, x)+\frac{1}{2}\left(\partial_{x} \zeta(t, x)\right)^{2} \leq-\delta .
$$

Since $\zeta$ is semiconvex, Alexandrov's theorem [1] (see also [28, Theorem 3.11]) ensures that such an expansion is valid at almost every $(t, x) \in U$. In particular there exists a sequence $\left(t_{k}, x_{k}\right) \rightarrow 0$ such that $\zeta$ is differentiable at $\left(t_{k}, x_{k}\right)$ and

$$
\partial_{t} \zeta\left(t_{k}, x_{k}\right)+\frac{1}{2}\left(\partial_{x} \zeta\left(t_{k}, x_{k}\right)\right)^{2} \leq-\delta .
$$

Up to extracting a subsequence we may assume that $\left(v_{k}, w_{k}\right):=\nabla \zeta\left(t_{k}, x_{k}\right)$ converges towards $(v, w) \in \mathbb{R}^{2}$ that satisfies

$$
v+\frac{1}{2} w^{2} \leq-\delta
$$

Moreover the convex function $\zeta+(2 r)^{-1}\left(\left(t-t_{k}\right)^{2}+\left(x-x_{k}\right)^{2}\right)$ lies above its tangent: for all $(t, x) \in U$ it holds

$$
\zeta(t, x)+\frac{1}{2 r}\left(\left(t-t_{k}\right)^{2}+\left(x-x_{k}\right)^{2}\right) \geq \zeta\left(t_{k}, x_{k}\right)+v_{k}\left(t-t_{k}\right)+w_{k}\left(x-x_{k}\right) .
$$

Passing to the limit yields (12) for $\zeta_{a}(t, x)=\zeta(0,0)+v t+w x$, and (11) follows from (13).

Step 2. For any height $\eta>0$ with

$$
\eta \ll \delta^{5} r
$$

(where the symbol $\ll$ denotes inequality up to a small universal constant), letting

$$
\widetilde{\zeta}(t, x):=\zeta_{a}(t, x)-\frac{1}{2 r}|(t, x)|^{2}-\frac{\delta}{2 r}|(t, x)|^{2}, \quad|(t, x)|^{2}=t^{2}+x^{2},
$$

and defining as in [12] the set

$$
\Omega_{\eta}:=B_{\rho} \cap\{\widetilde{\zeta}+\eta \geq h\},
$$

it holds

$$
B_{\eta / 3} \subset \Omega_{\eta} \subset B_{2(r \eta / \delta)^{1 / 2}} \subset \subset B_{\rho} .
$$

Since $h$ and $\zeta_{a}$ are 1-Lipschitz, in $B_{\eta / 3}$ we obtain

$$
\begin{aligned}
\widetilde{\zeta}+\eta-h & \geq \eta-2|(t, x)|-\frac{1+\delta}{2 r}|(t, x)|^{2} \\
& \geq \eta-\left(2+\frac{\eta}{3 r}\right)|(t, x)| \geq \eta-\left(2+\frac{\delta^{5}}{30}\right)|(t, x)| \\
& \geq \eta-3|(t, x)|>0,
\end{aligned}
$$


which implies $B_{\eta / 3} \subset \Omega_{\eta}$.

The strict inclusion $B_{2(r \eta / \delta)^{1 / 2}} \subset \subset B_{\rho}$ follows from (14) and $\rho \geq \delta^{2} r$ which imply

$$
2\left(\frac{r \eta}{\delta}\right)^{1 / 2} \ll \delta^{2} r<\rho
$$

Moreover since $h \geq \zeta$ in $B_{\rho}$ and (12) holds, in $B_{\rho} \backslash B_{2(r \eta / \delta)^{1 / 2}}$ we have

$$
h-\widetilde{\zeta} \geq \frac{\delta}{2 r}\left(t^{2}+x^{2}\right) \geq 2 \eta,
$$

which shows $\Omega_{\eta} \subset B_{2(r \eta / \delta)^{1 / 2}}$.

Step 3. Denoting by $\langle f\rangle$ the average of a function $f$ in $\Omega_{\eta}$ and assuming

$$
\eta \ll \delta^{3} r
$$

it holds

$$
\delta \leq\left\langle(u-\langle u\rangle)^{2}\right\rangle .
$$

Using the definition of $\widetilde{\zeta}$ in Step 2 together with (11), (16) and the fact that $\left|\partial_{x} \zeta_{a}\right| \leq 1$, in $B_{2(r \eta / \delta)^{1 / 2}}$ we find

$$
\begin{aligned}
\partial_{t} \widetilde{\zeta}+\frac{1}{2}\left(\partial_{x} \widetilde{\zeta}\right)^{2} & =\partial_{t} \zeta_{a}+\frac{1}{2}\left(\partial_{x} \zeta_{a}\right)^{2}-\frac{1+\delta}{r} t-2 \frac{1+\delta}{r} x \partial_{x} \zeta_{a}+\frac{(1+\delta)^{2}}{2 r^{2}} x^{2} \\
& \leq-\delta+C\left(\frac{\eta}{\delta^{3} r}\right)^{1 / 2} \delta \leq-\frac{\delta}{2}
\end{aligned}
$$

By (15) this holds in particular in $\Omega_{\eta}$ and therefore using Jensen's inequality we have

$$
-\frac{\delta}{2} \geq\left\langle\partial_{t} \widetilde{\zeta}\right\rangle+\frac{1}{2}\left\langle\left(\partial_{x} \widetilde{\zeta}\right)^{2}\right\rangle \geq\left\langle\partial_{t} \widetilde{\zeta}\right\rangle+\frac{1}{2}\left\langle\partial_{x} \widetilde{\zeta}\right\rangle^{2}
$$

Moreover since $u=\partial_{x} h$ and $(\widetilde{\zeta}+\eta-h)_{+}$has compact support in $B_{\rho}$ it holds

$$
\left\langle\partial_{x} \widetilde{\zeta}\right\rangle-\langle u\rangle=\frac{1}{\left|\Omega_{\eta}\right|} \int_{B_{\rho}} \partial_{x}\left[(\widetilde{\zeta}+\eta-h)_{+}\right]=0
$$

and similarly $\left\langle\partial_{t} \widetilde{\zeta}\right\rangle=\left\langle-u^{2} / 2\right\rangle$. This implies

$$
-\frac{\delta}{2} \geq-\frac{1}{2}\left\langle(u-\langle u\rangle)^{2}\right\rangle,
$$

and proves (17).

Step 4. It holds

$$
\left\langle(u-\langle u\rangle)^{2}\right\rangle^{2} \lesssim\left(\frac{\eta}{\delta r}\right)^{1 / 2}+\frac{\left\|\mu_{+}\right\|}{\eta},
$$


where the symbol $\lesssim$ stands for inequality up to a universal constant.

The argument relies as in [12] on a quantification of Tartar's application of the div-curl lemma to equations of Burgers type [32]. By Hölder's inequality and [12, Proposition 3.2] we have

$$
\begin{aligned}
\left\langle(u-\langle u\rangle)^{2}\right\rangle^{2} & \leq\left\langle(u-\langle u\rangle)^{4}\right\rangle \\
& \lesssim\left\langle\left(\begin{array}{c}
-\frac{u^{2}}{2} \\
u
\end{array}\right) \cdot\left(\begin{array}{c}
u^{2} / 2 \\
u^{3} / 3
\end{array}\right)\right\rangle-\left\langle\left(\begin{array}{c}
-\frac{u^{2}}{2} \\
u
\end{array}\right)\right\rangle \cdot\left\langle\left(\begin{array}{c}
u^{2} / 2 \\
u^{3} / 3
\end{array}\right)\right\rangle \\
& =\left\langle\left(\begin{array}{c}
\partial_{t} h \\
\partial_{x} h
\end{array}\right) \cdot\left(\begin{array}{c}
u^{2} / 2 \\
u^{3} / 3
\end{array}\right)\right\rangle-\left\langle\left(\begin{array}{c}
\partial_{t} h \\
\partial_{x} h
\end{array}\right)\right\rangle \cdot\left\langle\left(\begin{array}{c}
u^{2} / 2 \\
u^{3} / 3
\end{array}\right)\right\rangle
\end{aligned}
$$

Recalling (18) and its counterpart for the $t$-derivative, we deduce

$$
\begin{aligned}
\left\langle(u-\langle u\rangle)^{2}\right\rangle^{2} \lesssim & \left\langle\left(\begin{array}{c}
\partial_{t}(h-\widetilde{\zeta}-\eta) \\
\partial_{x}(h-\widetilde{\zeta}-\eta)
\end{array}\right) \cdot\left(\begin{array}{c}
u^{2} / 2 \\
u^{3} / 3
\end{array}\right)\right\rangle \\
& +\left\langle\left(\begin{array}{c}
\partial_{t} \widetilde{\zeta}-\left\langle\partial_{t} \widetilde{\zeta}\right\rangle \\
\partial_{x} \widetilde{\zeta}-\left\langle\partial_{x} \widetilde{\zeta}\right\rangle
\end{array}\right) \cdot\left(\begin{array}{c}
u^{2} / 2 \\
u^{3} / 3
\end{array}\right)\right\rangle \\
\leq & \frac{1}{\left|\Omega_{\eta}\right|} \int_{\Omega_{\eta}}(\widetilde{\zeta}+\eta-h) d \mu+\frac{2}{r} \operatorname{diam}\left(\Omega_{\eta}\right)
\end{aligned}
$$

For the last inequality we used the fact that, $\zeta_{a}$ being affine, we have

$$
\nabla \widetilde{\zeta}-\langle\nabla \widetilde{\zeta}\rangle=-\frac{1+\delta}{r}\left(\begin{array}{c}
t \\
x
\end{array}\right)
$$

Since in $\Omega_{\eta}$ it holds $0 \leq \widetilde{\zeta}+\eta-h \leq \eta+\zeta-h \leq \eta$, we find

$$
\left\langle(u-\langle u\rangle)^{2}\right\rangle^{2} \lesssim \frac{\eta}{\left|\Omega_{\eta}\right|}\left\|\mu_{+}\right\|+\frac{2}{r} \operatorname{diam}\left(\Omega_{\eta}\right) .
$$

Using the inclusions (15) satisfied by $\Omega_{\eta}$ we obtain (19).

Step 5. Conclusion.

We choose $\eta=(\delta r)^{1 / 3}\left\|\mu_{+}\right\|^{2 / 3}$ in order to balance the two terms on the righthand side of (19). Since

$$
(\delta r)^{1 / 3}\left\|\mu_{+}\right\|^{2 / 3} \leq \frac{1}{C^{2 / 3}} \delta^{5} r,
$$

the restrictions (14) and (16) on $\eta$ are indeed satisfied provided $C$ is large enough. Moreover combining (17) and (19) with the smallness assumption (10) on $\mu_{+}$, we obtain

$$
\delta^{2} \lesssim\left(\frac{\left\|\mu_{+}\right\|}{\delta r}\right)^{1 / 3} \lesssim \frac{1}{C^{1 / 3}} \delta^{2},
$$

and therefore the desired contradiction for large enough $C$. 
Proof of Proposition 7. Note that since $h \leq \bar{h}$ we only need to estimate $(h-\bar{h})$ from below. Given $\rho \in(0,1)$ we consider the sup-convolution

$$
\bar{h}_{\rho}(t, x)=\sup _{(s, y) \in Q}\left\{\bar{h}(s, y)-\frac{1}{2 \rho}\left((t-s)^{2}+(x-y)^{2}\right)\right\} .
$$

As a supremum of $(1 / \rho)$-semiconvex functions this function $\bar{h}_{\rho}$ is $(1 / \rho)$-semiconvex. We also introduce parameters $\delta \in(0,1), H, M \geq 1$ and define

$$
\zeta(t, x)=\bar{h}_{\rho}(t, x)-\delta t-\frac{M}{2}\left(\left(t-t_{1}\right)_{+}\right)^{2}-H \rho,
$$

so that $\zeta$ is $(1 / r)$-semiconvex with $1 / r=1 / \rho+M$. We want to use Lemma 8 to deduce that $h \geq \zeta$ in $Q$ and from there obtain the desired lower bound on $(h-\bar{h})$. We split the proof in the following way : in Step 1 we prove that $\zeta$ is a viscosity subsolution as in Lemma 8; then we show that $h \geq \zeta$ near the boundary, dealing with the parabolic boundary $\partial_{0} Q$ in Step 2 and the remaining boundary in Step 3 ; in Step 4 we check that the Lipschitz constant of $\zeta$ depends only on $L$ and $t_{1}$ in the relevant region; eventually in Step 5 we apply Lemma 8 and optimize the choices of $\rho$ and $\delta$ in order to conclude.

Step 1. For $\rho \ll 1 / L$, the function $\zeta$ is a viscosity subsolution of

$$
\partial_{t} \zeta+\frac{1}{2}\left(\partial_{x} \zeta\right)^{2} \leq-\delta \quad \text { in } \widetilde{Q}:=Q \cap\{\operatorname{dist}(\cdot, \partial Q)>4 L \rho\}
$$

It suffices to show that $\bar{h}_{\rho}$ is a viscosity subsolution of

$$
\partial_{t} \bar{h}_{\rho}+\frac{1}{2}\left(\partial_{x} \bar{h}_{\rho}\right)^{2} \leq 0 \quad \text { in } \widetilde{Q}
$$

The fact that sup convolution preserves the viscosity subsolution property is well-known, see e.g. [7, Lemma A.5]. For the convenience of the reader we provide a proof of $(22)$ in our setting. Let $\varphi(t, x)$ be a smooth function such that $\left(\bar{h}_{\rho}-\varphi\right)$ attains its maximum at $\left(t_{0}, x_{0}\right) \in \widetilde{Q}$, and assume w.l.o.g. that $\varphi\left(t_{0}, x_{0}\right)=\bar{h}_{\rho}\left(t_{0}, x_{0}\right)$. For any $(s, y) \in U$ with

$$
d:=\left|(s, y)-\left(t_{0}, x_{0}\right)\right|=\sqrt{\left(t_{0}-s\right)^{2}+\left(x_{0}-y\right)^{2}} \geq 2 L \rho,
$$

since by $(8)$ we have $\operatorname{Lip}(\bar{h}) \leq L$, it holds

$$
\begin{aligned}
\bar{h}(s, y)-\frac{1}{2 \rho} d^{2} & \leq \bar{h}\left(t_{0}, x_{0}\right)+\left(L-\frac{d}{2 \rho}\right) d \\
& \leq \bar{h}\left(t_{0}, x_{0}\right) \leq \bar{h}_{\rho}\left(t_{0}, x_{0}\right) .
\end{aligned}
$$

Hence the supremum in the definition of $\bar{h}_{\rho}\left(t_{0}, x_{0}\right)$ is attained at some $\left(s_{0}, y_{0}\right) \in$ $B_{2 L \rho}\left(t_{0}, x_{0}\right) \subset Q$, and

$$
\varphi\left(t_{0}, x_{0}\right)=\bar{h}_{\rho}\left(t_{0}, x_{0}\right)=\bar{h}\left(s_{0}, y_{0}\right)-\frac{1}{2 \rho}\left(\left(t_{0}-s_{0}\right)^{2}+\left(x_{0}-y_{0}\right)^{2}\right) .
$$


Moreover since $\left(\bar{h}_{\rho}-\varphi\right)$ is maximal at $\left(t_{0}, x_{0}\right)$ with value zero, it holds

$$
\bar{h}(s, y)-\frac{1}{2 \rho}\left((t-s)^{2}+(x-y)^{2}\right) \leq \varphi(t, x) \quad \forall(t, x),(s, y) \in Q .
$$

In particular for all $(s, y) \in B_{2 L \rho}\left(s_{0}, y_{0}\right) \subset Q$ we may choose

$$
(t, x)=\left(s-s_{0}+t_{0}, y-y_{0}+x_{0}\right) \in B_{2 L \rho}\left(t_{0}, x_{0}\right) \subset Q,
$$

in (24) and obtain

$$
\bar{h}(s, y) \leq \varphi\left(s-s_{0}+t_{0}, y-y_{0}+x_{0}\right)+\frac{1}{2 \rho}\left(\left(t_{0}-s_{0}\right)^{2}+\left(x_{0}-y_{0}\right)^{2}\right)=: \psi(s, y) .
$$

Moreover (23) ensures $\psi\left(s_{0}, y_{0}\right)=\bar{h}\left(s_{0}, y_{0}\right)$, hence $\bar{h}-\psi$ has a local maximum at $\left(s_{0}, y_{0}\right)$. Since $\bar{h}$ is a viscosity solution we deduce that

$$
\partial_{t} \varphi\left(t_{0}, x_{0}\right)+\frac{1}{2}\left(\partial_{x} \varphi\left(t_{0}, x_{0}\right)\right)^{2}=\partial_{t} \psi\left(s_{0}, y_{0}\right)+\frac{1}{2}\left(\partial_{x} \psi\left(s_{0}, y_{0}\right)\right)^{2} \leq 0,
$$

which proves (22).

Step 2. Provided $H$ is large enough (depending only on $L$ ) it holds

$$
\zeta \leq h \quad \text { in } Q \cap\left\{\operatorname{dist}\left(\cdot, \partial_{0} Q\right) \leq 4 L \rho+\delta^{2} r\right\} .
$$

The definition of $\bar{h}_{\rho}$ implies

$$
\bar{h}_{\rho}(t, x) \leq \bar{h}(t, x)+\frac{L^{2}}{2} \rho .
$$

By definition of $\zeta$ this yields

$$
\zeta(t, x)-h(t, x) \leq-\frac{M}{2}\left(\left(t-t_{1}\right)_{+}\right)^{2}-H \rho+\frac{L^{2}}{2} \rho+\bar{h}(t, x)-h(t, x),
$$

so that by $\bar{h}=h$ on $\partial_{0} Q$ and the Lipschitz continuities of $h$ and $\bar{h}(8)$ we obtain

$$
\zeta(t, x)-h(t, x) \leq-\frac{M}{2}\left(\left(t-t_{1}\right)_{+}\right)^{2}-H \rho+\frac{L^{2}}{2} \rho+2 L \operatorname{dist}\left((t, x), \partial_{0} Q\right) .
$$

Therefore if $\operatorname{dist}\left((t, x), \partial_{0} Q\right) \leq 4 L \rho+\delta^{2} r \leq 5 L \rho$ we have

$$
\begin{aligned}
\zeta(t, x)-h(t, x) & \leq \bar{h}_{\rho}(t, x)-\bar{h}(t, x)+\bar{h}(t, x)-h(t, x)-H \rho \\
& \leq\left(\frac{L^{2}}{2}+10 L^{2}-H\right) \rho,
\end{aligned}
$$

and it suffices to choose $H \geq 11 L^{2}$.

Step 3. Provided $M$ is large enough (depending only on $L$ and $t_{1}$ ) it holds

$$
\zeta \leq h \quad \text { in } Q \cap\left\{t \geq\left(1+t_{1}\right) / 2\right\} .
$$


For $t \geq\left(1+t_{1}\right) / 2$ we have by $(25)$

$$
\begin{aligned}
\zeta(t, x)-h(t, x) & \leq \frac{L^{2}}{2} \rho+2 L-\frac{M}{8}\left(1-t_{1}\right)^{2} \\
& \leq \frac{L^{2}}{2}+2 L-\frac{M}{8}\left(1-t_{1}\right)^{2},
\end{aligned}
$$

and it suffices to choose $M \geq\left(4 L^{2}+16 L\right) /\left(1-t_{1}\right)^{2}$.

Step 4. We have

$$
\operatorname{Lip}(\zeta) \leq 2 L+2 M \quad \text { in } \widetilde{Q}
$$

where $\widetilde{Q}=Q \cap\{\operatorname{dist}(\cdot, \partial Q)>4 L \rho\}$ as in Step 1 .

It was shown in Step 1 that for $\left(t_{0}, x_{0}\right)$ in $\widetilde{Q}$, the supremum in the definition of $\bar{h}_{\rho}\left(t_{0}, x_{0}\right)$ is attained at some $\left(s_{0}, y_{0}\right) \in B_{2 L \rho}\left(t_{0}, x_{0}\right)$. It follows that for any small $(t, x)$ we have

$$
\begin{aligned}
\bar{h}_{\rho}\left(t_{0}, x_{0}\right)- & \bar{h}_{\rho}\left(t_{0}+t, x_{0}+x\right) \\
= & \bar{h}\left(s_{0}, y_{0}\right)-\frac{1}{2 \rho}\left|\left(t_{0}-s_{0}, x_{0}-y_{0}\right)\right|^{2} \\
& -\sup _{(s, y) \in Q}\left\{\bar{h}(s, y)-\frac{1}{2 \rho}\left|\left(t_{0}+t-s, x_{0}+x-y\right)\right|^{2}\right\} \\
\leq & \frac{1}{2 \rho}\left(2\left(t_{0}-s_{0}\right) t+2\left(x_{0}-y_{0}\right) x+|(t, x)|^{2}\right) \\
\leq & 2 L|(t, x)|+\frac{1}{2 \rho}|(t, x)|^{2} .
\end{aligned}
$$

This implies $\left|\nabla \bar{h}_{\rho}\right| \leq 2 L$ in $\widetilde{Q}$. Therefore in $\widetilde{Q}$ it holds

$$
\operatorname{Lip}(\zeta) \leq 2 L+\delta+M
$$

which concludes the proof of Step 4 since $\delta \leq 1$ and $M \geq 1$.

Step 5. Conclusion.

Recalling (21) and Step 4, Lemma 8 ensures the existence of a constant $C>0$ depending through $\operatorname{Lip}(h)$ and $\operatorname{Lip}(\zeta)$ only on $L$ and $t_{1}$, such that if $C\left\|\mu_{+}\right\| \leq \delta^{7} r$ then the minimum of $(h-\zeta)$ in $\bar{Q}$ cannot be attained at any $x \in \widetilde{Q}$ such that $B_{\delta^{2} r}(x) \subset \widetilde{Q}$. Moreover if $4 L \rho+\delta^{2} r \leq\left(1-t_{1}\right) / 2$ then by Steps 2 and 3 , at any $x \in \widetilde{Q}$ such that $B_{\delta^{2} r}(x)$ is not contained in $\widetilde{Q}$ it must hold $h-\zeta \geq 0$.

Since $\delta^{2} r \leq \rho$ we deduce that if $\rho \leq \rho_{0}:=\left(1-t_{1}\right) /(8 L+2)$ and $C\left\|\mu_{+}\right\| \leq \delta^{7} r$ then $h-\zeta \geq 0$ in $\bar{Q}$. Hence for $t \leq t_{1}$ it holds

$$
\begin{aligned}
h-\bar{h} & \geq \zeta-\bar{h} \\
& =\bar{h}_{\rho}-\bar{h}-\delta t-H \rho \\
& \geq-H \rho-\delta,
\end{aligned}
$$

and therefore

$$
\sup _{Q \cap\left\{t \leq t_{1}\right\}}|h-\bar{h}| \leq H \rho+\delta .
$$


Choosing $\delta=\rho$ and recalling that $r=\rho /(1+\rho M) \geq \rho /(1+M) \geq \rho /(2 M)$ we conclude that

$$
2 M C\left\|\mu_{+}\right\| \leq \rho^{8} \Longrightarrow \sup _{Q \cap\left\{t \leq t_{1}\right\}}|h-\bar{h}| \leq(1+H) \rho .
$$

If $\left\|\mu_{+}\right\| \leq \rho_{0}^{8} /(2 M C)$ we can apply this to $\rho=\left(2 M C\left\|\mu_{+}\right\|\right)^{1 / 8}$ to finish the proof. If $\left\|\mu_{+}\right\|>\rho_{0}^{8} /(2 M C)$ we can simply invoke the fact that $|h-\bar{h}|$ is bounded by a constant depending only on $L$.

\section{Proofs of Theorems 5 and 2}

In this section we use the symbol $\lesssim$ to denote inequality up to a constant depending only on $\|u\|_{\infty}$.

Proof of Theorem 5. Let $h$ be the Lipschitz solution of (7) such that $u=\partial_{x} h$ and $-u^{2} / 2=\partial_{t} h$, let $\bar{h}$ be as in $\S 2$ the viscosity solution of (7) in $Q_{1}$ with $\bar{h}=h$ on the parabolic boundary $\partial_{0} Q_{1}$. By Proposition 7 it holds

$$
\sup _{Q_{7 / 8}}|\bar{h}-h| \lesssim \mu_{+}\left(Q_{1}\right)^{\frac{1}{8}}
$$

This estimate tells us that $u$ is close to the entropy solution $\zeta=\partial_{x} \bar{h}$ in a weak sense. The rest of the proof consists in transforming this weak estimate into the desired $L^{1}$-estimate: in a first step we quantify the compactness of solutions of (1) satisfying (4), and in a second step we use this quantitative compactness and a standard interpolation argument to conclude.

Step 1. We show that

$$
\sup _{|\xi| \leq r / 4} \int_{Q_{3 / 4}}(u(\cdot+\xi)-u)^{4} \lesssim r\left(1+|\mu|\left(Q_{1}\right)\right)+\frac{1}{r} \mu_{+}\left(Q_{1}\right)^{\frac{1}{8}}
$$

for all $r \in(0,1 / 8)$.

The proof relies on a "div-curl" argument used also in [16]. First we use the Galilean invariance of (1): for any constant $c \in \mathbb{R}$ the function $\tilde{u}=u-c$ satisfies

$$
\begin{gathered}
\left(\partial_{t}+c \partial_{x}\right) \tilde{u}+\partial_{x} \frac{\tilde{u}^{2}}{2}=0 \\
\left(\partial_{t}+c \partial_{x}\right) \frac{\tilde{u}^{2}}{2}+\partial_{x} \frac{\tilde{u}^{3}}{3}=\mu,
\end{gathered}
$$

and $\tilde{u}=\partial_{x} \tilde{h},-\tilde{u}^{2} / 2=\left(\partial_{t}+c \partial_{x}\right) \tilde{h}$, where

$$
\tilde{h}=h-c x+\frac{1}{2} c^{2} t .
$$


We infer that for any $z_{0} \in Q_{3 / 4}$ it holds

$$
\begin{aligned}
\frac{1}{12} \tilde{u}^{4}= & \left(\begin{array}{c}
\frac{1}{2} \tilde{u}^{2} \\
\frac{1}{3} \tilde{u}^{3}
\end{array}\right) \cdot\left(\begin{array}{c}
\left(\partial_{t}+c \partial_{x}\right)\left(\tilde{h}-\tilde{h}\left(z_{0}\right)\right) \\
\partial_{x}\left(\tilde{h}-\tilde{h}\left(z_{0}\right)\right)
\end{array}\right) \\
= & \left(\begin{array}{c}
\partial_{t}+c \partial_{x} \\
\partial_{x}
\end{array}\right) \cdot\left[\left(\tilde{h}-\tilde{h}\left(z_{0}\right)\right)\left(\begin{array}{c}
\frac{1}{2}(u-c)^{2} \\
\frac{1}{3}(u-c)^{3}
\end{array}\right)\right] \\
& -\left(\tilde{h}-\tilde{h}\left(z_{0}\right)\right) \mu .
\end{aligned}
$$

We multiply this identity by a smooth cut-off function at scale $r \in(0,1 / 8)$ and deduce, restricting ourselves to $|c| \lesssim 1$ and recalling that $\operatorname{Lip}(\bar{h}) \lesssim 1$,

$$
\int_{Q_{r / 2}\left(z_{0}\right)} \tilde{u}^{4} \lesssim \frac{1}{r} \int_{Q_{r}\left(z_{0}\right)}\left|\tilde{h}-\tilde{h}\left(z_{0}\right)\right|+r|\mu|\left(Q_{r}\left(z_{0}\right)\right) .
$$

To estimate the first term on the right-hand side we wish to pass from $h$ to the viscosity solution $\bar{h}$, since it is semi-concave and in particular twice differentiable almost everywhere. In fact in $Q_{7 / 8}$ the second derivative of $\bar{h}$ in any direction is bounded from above by a universal constant [25, Theorem 13.1] and therefore, in conjunction with $\operatorname{Lip}(\bar{h}) \lesssim 1$,

$$
\int_{Q_{7 / 8}}\left|\nabla^{2} \bar{h}\right| \lesssim 1
$$

We write

$$
\begin{aligned}
\int_{Q_{r}\left(z_{0}\right)}\left|\tilde{h}-\tilde{h}\left(z_{0}\right)\right| \lesssim & r^{2} \sup _{Q_{7 / 8}}|h-\bar{h}| \\
& +\int_{Q_{r}\left(z_{0}\right)}\left|\bar{h}-\left(\bar{h}\left(z_{0}\right)+c\left(x-x_{0}\right)-\frac{1}{2} c^{2}\left(t-t_{0}\right)\right)\right| .
\end{aligned}
$$

Next we assume that $\bar{h}$ is differentiable at $z_{0}$ (which is the case for almost every $z_{0}$ ) and choose $c=c\left(z_{0}\right)=\partial_{x} \bar{h}\left(z_{0}\right)$, so that $\partial_{t} \bar{h}\left(z_{0}\right)=-c^{2} / 2$ and, using also (26) the above turns into

$$
\begin{aligned}
\int_{Q_{r}\left(z_{0}\right)}\left|\tilde{h}-\tilde{h}\left(z_{0}\right)\right| \lesssim & r^{2} \sup _{Q_{7 / 8}}|h-\bar{h}| \\
& +\int_{Q_{r}\left(z_{0}\right)}\left|\bar{h}(z)-\left(h\left(z_{0}\right)+\nabla \bar{h}\left(z_{0}\right) \cdot\left(z-z_{0}\right)\right)\right| d z \\
\lesssim & r^{2}\left[\mu_{+}\left(Q_{1}\right)\right]^{1 / 8}+r^{2} \int_{0}^{1} \int_{Q_{r}}\left|\nabla^{2} \bar{h}\left(z_{0}+s \tilde{z}\right)\right| d \tilde{z} d s .
\end{aligned}
$$

Plugging this back into (28) we deduce that for $|\xi| \leq r / 4$ it holds

$$
\begin{aligned}
\int_{Q_{r / 4}\left(z_{0}\right)}(u(\cdot+\xi)-u)^{4} \lesssim & \int_{Q_{r / 2}\left(z_{0}\right)}\left(u-c\left(z_{0}\right)\right)^{4} \\
\lesssim & r|\mu|\left(Q_{r}\left(z_{0}\right)\right)+r\left[\mu_{+}\left(Q_{1}\right)\right]^{1 / 8} \\
& +r \int_{0}^{1} \int_{Q_{r}}\left|\nabla^{2} \bar{h}\left(z_{0}+s \tilde{z}\right)\right| d \tilde{z} d s
\end{aligned}
$$


Integrating over $z_{0} \in Q_{3 / 4}$, dividing by $r^{2}$, and recalling (29) we obtain (27).

Step 2. Conclusion.

Recall that $\zeta$ is the entropy solution of (1) given by $\zeta=\partial_{x} \bar{h}$. Next we use the compactness $(27)$ to turn (26) into an $L^{1}$-estimate on

$$
u-\zeta=\partial_{x}(h-\bar{h}) .
$$

We introduce a smooth kernel $\varphi(z)$ with compact support in $Q_{1}$ and unit integral and define

$$
u_{r}=u \star \varphi_{r}, \quad \zeta_{r}=\zeta \star \varphi_{r}, \quad \text { where } \varphi_{r}(z)=r^{-2} \varphi(z / r) .
$$

From (27) we deduce that for $r \in(0,1 / 8)$ it holds

$$
\int_{Q_{3 / 4}}\left|u-u_{r}\right|^{4} \lesssim \varepsilon\left(1+|\mu|\left(Q_{1}\right)\right)+\frac{1}{r} \mu_{+}\left(Q_{1}\right)^{\frac{1}{8}}
$$

Moreover, (29) implies that

$$
\int_{Q_{3 / 4}}\left|\zeta-\zeta_{r}\right|^{4} \lesssim r^{4}
$$

The combination yields

$$
\begin{aligned}
\int_{Q_{3 / 4}}|u-\zeta|^{4} & \lesssim r^{4}+r\left(1+|\mu|\left(Q_{1}\right)\right)+\frac{1}{r} \mu_{+}\left(Q_{1}\right)^{\frac{1}{8}}+\int_{Q_{3 / 4}}\left|(h-\bar{h}) \star \partial_{x} \varphi_{r}\right|^{4} \\
& \lesssim r^{4}+r\left(1+|\mu|\left(Q_{1}\right)\right)+\frac{1}{r} \mu_{+}\left(Q_{1}\right)^{\frac{1}{8}}+\frac{1}{r^{4}} \mu_{+}\left(Q_{1}\right)^{\frac{1}{2}}
\end{aligned}
$$

where we used (26) in the second step. We choose $r=\left[\mu_{+}\left(Q_{1}\right)\right]^{1 / 16}\left(1+|\mu|\left(Q_{1}\right)\right)^{-1 / 5}$, which is admissible since without loss of generality $\mu+\left(Q_{1}\right) \ll 1$, to find our conclusion

$$
\int_{Q_{3 / 4}}|u-\zeta|^{4} \lesssim\left(1+|\mu|\left(Q_{1}\right)\right)^{4 / 5}\left[\mu_{+}\left(Q_{1}\right)\right]^{1 / 16} .
$$

Proof of Theorem 2. Step 1. If $u$ is as in Theorem 5, then for any $\theta \in(0,3 / 4)$ it holds

$$
f_{|t| \leq \theta} f_{|x| \leq \theta} f_{|y| \leq \theta}|u(t, x)-u(t, y)| d x d y d t \lesssim \theta+\frac{1}{\theta^{3}} \min \left(1,|\mu|\left(Q_{1}\right)^{\frac{1}{64}}\right) .
$$

To prove this estimate we apply Theorem 5 to $u$ and to $-u(-t, x)$ and deduce the existence of an entropy solution $\bar{\zeta}$ and an anti-entropy solution $\underline{\zeta}$ of (1) in $Q_{1}$ with

$$
\int_{Q_{3 / 4}}|u-\bar{\zeta}|+\int_{Q_{3 / 4}}|u-\underline{\zeta}| \lesssim\left(1+|\mu|\left(Q_{1}\right)\right)^{\frac{1}{5}}|\mu|\left(Q_{1}\right)^{\frac{1}{64}} .
$$


Since $\bar{\zeta}$ is an entropy solution and $\underline{\zeta}$ an anti-entropy solution it holds by Oleinik's principle

$$
\partial_{x} \bar{\zeta} \lesssim 1 \quad \text { and } \quad-\partial_{x} \underline{\zeta} \lesssim 1 \quad \text { in } Q_{3 / 4}
$$

Combining these facts we compute

$$
\begin{aligned}
f_{|t| \leq \theta} f_{|x| \leq \theta} f_{|y| \leq \theta}|u(t, x)-u(t, y)| \lesssim & \frac{1}{\theta^{3}} \int_{Q_{3 / 4}}|u-\bar{\zeta}|+\frac{1}{\theta^{3}} \int_{Q_{3 / 4}}|u-\underline{\zeta}| \\
& +f_{|t| \leq \theta} \frac{1}{\theta^{2}} \iint_{\theta>x>y>-\theta}(\bar{\zeta}(t, x)-\bar{\zeta}(t, y))_{+} \\
& +f_{|t| \leq \theta} \frac{1}{\theta^{2}} \iint_{-\theta<x<y<\theta}(\underline{\zeta}(t, x)-\underline{\zeta}(t, y))_{+} \\
\lesssim & \frac{1}{\theta^{3}}\left(1+|\mu|\left(Q_{1}\right)^{\frac{1}{5}}|\mu|\left(Q_{1}\right)^{\frac{1}{64}}+\theta .\right.
\end{aligned}
$$

This proves (30) if $|\mu|\left(Q_{1}\right) \leq 1$, and for $|\mu|\left(Q_{1}\right) \geq 1$ the left-hand side of (30) is $\lesssim 1$ so that $(30)$ holds.

Step 2. If $u$ is as in Theorem 2 and $z_{0}=\left(t_{0}, x_{0}\right) \in \Omega_{\alpha, K}$ then it holds

$$
D(r):=f_{\left|t-t_{0}\right| \leq r} f_{\left|x-x_{0}\right| \leq r} f_{\left|y-x_{0}\right| \leq r}|u(t, x)-u(t, y)| d x d y d t \lesssim C\left(K, d\left(z_{0}\right), \alpha\right) r^{\frac{\alpha}{\alpha+256}}
$$

for all $r \in\left(0, d\left(z_{0}\right)\right)$.

We assume without loss of generality $K \geq 1$. For any $\rho \in\left(0, d\left(z_{0}\right) \wedge K^{-1 / \alpha}\right)$ we apply Step 1 to $z \mapsto u\left(\rho\left(z-z_{0}\right)\right)$ and obtain

$$
D\left(\frac{3}{4} \theta \rho\right) \lesssim \theta+\frac{1}{\theta^{3}} K^{\frac{1}{64}} \rho^{\frac{\alpha}{64}} \quad \text { for all } \theta \in(0,1) .
$$

We choose $\theta=\left(K^{\frac{1}{64}} \rho^{\frac{\alpha}{64}}\right)^{\frac{1}{4}}$ to balance the two terms and set $r=\frac{3}{4} \theta \rho=$ $\frac{3}{4} K^{\frac{1}{256}} \rho^{\frac{256+\alpha}{256}}$. This yields

$$
D(r) \lesssim K^{\frac{1}{\alpha+256}} r^{\frac{\alpha}{\alpha+256}} .
$$

This is valid for $r \leq r_{0}\left(K, d\left(z_{0}\right)\right)$ due to the constraints on $\rho$, and for $r \geq r_{0}$ we have $D(r) \lesssim\left(r / r_{0}\right)^{\frac{\alpha}{\alpha+256}}$

Step 3. From Step 2 we have the desired regularity in the space variable, and it remains to use the equation (1) to transfer it to the time variable. We sketch here the standard argument.

We use coordinates in which $z_{0}=0$, we fix a smooth cut-off function $\eta(x)$, set $\eta_{r}(x)=r^{-1} \eta\left(r^{-1} x\right)$ and notice that

$$
\frac{d}{d t}\left[\int u(t, x) \eta_{r}(x) d x\right]=\frac{1}{2} \int u^{2}(t, x)\left(\eta_{r}\right)^{\prime}(x) d x .
$$


In particular $t \mapsto \int u(t, x) \eta_{r}(x) d x$ is Lipschitz, and it holds

$$
\begin{gathered}
\int(u(s, x)-u(t, x)) \eta_{r}(x) d x=\frac{s-t}{2} \int_{0}^{1} \int u^{2}(\tau s+(1-\tau) t, x)\left(\eta_{r}\right)^{\prime}(x) d x d \tau \\
=\frac{s-t}{2 r} \int_{0}^{1} \int\left(u^{2}(\tau s+(1-\tau) t, x)-u^{2}(\tau s+(1-\tau) t, y)\right)\left(\eta^{\prime}\right)_{r}(x) d x d \tau
\end{gathered}
$$

where we used the fact that $\eta^{\prime}$ has zero average and where $y$ can be choosen arbitrarily. Hence together with $|u| \lesssim 1$ we obtain from averaging over $|t| \leq r$, $|s| \leq r$ and $|y| \leq r$

$$
f_{|s| \leq r} f_{|t| \leq r}\left|\int(u(s, x)-u(t, x)) \eta_{r}(x) d x\right| \lesssim D(r) .
$$

The conclusion then follows from Step 2.

\section{A Lipschitz estimate for the viscosity solu- tion}

Let $h \in W^{1, \infty}(Q)$ solve (7) almost everywhere. The viscosity solution $\bar{h} \in$ $W^{1, \infty}(Q)$ of $(7)$ with $\bar{h}=h$ on $\partial_{0} Q$ is given $[25, \S 11]$ by the Hopf-Lax formula

$$
\bar{h}(t, x)=\inf \left\{h(s, y)+\frac{(x-y)^{2}}{2(t-s)}:(s, y) \in \partial_{0} Q, s<t\right\} .
$$

Note that for $(t, x) \in Q$ the infimum is attained. Let $L:=\left\|\partial_{x} h\right\|_{L^{\infty}(Q)}$, so that the initial data $h(0, \cdot)$ has Lipschitz constant $\leq L$ and the boundary data $h(\cdot, 0)$ and $h(\cdot, 1)$ have Lipschitz constants $\leq L^{2} / 2$.

Lemma 9. It holds $\left|\partial_{x} \bar{h}\right| \leq L$ a.e.

Proof. Let $\left(t_{0}, x_{0}\right) \in Q$ and denote by $\left(s_{0}, y_{0}\right)$ a point at which the infimum defining $\bar{h}\left(t_{0}, x_{0}\right)$ is attained. Then for any small $x$ it holds

$$
\begin{aligned}
\bar{h}\left(t_{0}, x_{0}+x\right)-\bar{h}\left(t_{0}, x_{0}\right) & =\bar{h}\left(t_{0}, x_{0}+x\right)-h\left(s_{0}, y_{0}\right)-\frac{\left(x_{0}-y_{0}\right)^{2}}{2\left(t_{0}-s_{0}\right)} \\
& \leq \frac{\left(x_{0}+x-y_{0}\right)^{2}}{2\left(t_{0}-s_{0}\right)}-\frac{\left(x_{0}-y_{0}\right)^{2}}{2\left(t_{0}-s_{0}\right)} \\
& =\frac{x_{0}-y_{0}}{t_{0}-s_{0}} x+\frac{1}{2\left(t_{0}-s_{0}\right)} x^{2},
\end{aligned}
$$

so that $\left|\partial_{x} \bar{h}\left(t_{0}, x_{0}\right)\right| \leq\left|x_{0}-y_{0}\right| /\left(t_{0}-s_{0}\right)$ and to prove (8) it suffices to show that the infimum defining $\bar{h}\left(t_{0}, x_{0}\right)$ is attained at some $\left(s_{0}, y_{0}\right)$ with

$$
\frac{\left|x_{0}-y_{0}\right|}{t_{0}-s_{0}} \leq L
$$


We show that for any $(s, y) \in \partial_{0} Q \cap\left\{s<t_{0}\right\}$ with

$$
\frac{\left|x_{0}-y\right|}{t_{0}-s}>L
$$

there exists $(\tilde{s}, \tilde{y}) \in \partial_{0} Q \cap\left\{s<t_{0}\right\}$ satisfying

$$
\begin{aligned}
& \frac{\left|x_{0}-\tilde{y}\right|}{t_{0}-\tilde{s}}<\frac{\left|x_{0}-y\right|}{t_{0}-s} \\
\text { and } \quad & h\left(s_{0}, \tilde{y}\right)+\frac{\left(x_{0}-\tilde{y}\right)^{2}}{2\left(t_{0}-\tilde{s}\right)} \leq h\left(s_{0}, y\right)+\frac{\left(x_{0}-y\right)^{2}}{2\left(t_{0}-s\right)},
\end{aligned}
$$

which proves (31).

There are two cases to consider, depending on which part of the parabolic boundary $(s, y)$ belongs to.

Case $1:(s, y) \in\{0\} \times[0,1]$. We look for $(\tilde{s}, \tilde{y})$ defined through $\tilde{s}=0$ and

$$
\frac{x_{0}-\tilde{y}}{t_{0}}=(1-\varepsilon) D, \quad D:=\frac{x_{0}-y}{t_{0}},
$$

for some small $\epsilon>0$, so that (33) is satisfied. On the other hand since $h(0, \cdot)$ has Lipschitz constant $\leq L$, to show (34) it suffices to establish

$$
L|\tilde{y}-y| \leq \frac{\left(x_{0}-y\right)^{2}-\left(x_{0}-\tilde{y}\right)^{2}}{2 t_{0}} \Longleftrightarrow \frac{L}{D} \leq\left(1-\frac{1}{2} \varepsilon\right),
$$

which is satisfied for small enough $\varepsilon$ since (32) amounts to $|D|>L$.

Case $2:(s, y) \in(0,1) \times\{0,1\}$. We assume $y=0$, the case $y=1$ being similar. We look for $(\tilde{s}, \tilde{y})$ defined through $\tilde{y}=0$ and

$$
\frac{x_{0}}{t_{0}-\tilde{s}}=(1-\varepsilon) D, \quad D:=\frac{x_{0}}{t_{0}-s},
$$

for some small $\epsilon>0$, so that (33) is satisfied. On the other hand since $h(1, \cdot)$ has Lipschitz constant $\leq L^{2} / 2$, to show (34) it suffices to establish

$$
\frac{L^{2}}{2}|s-\tilde{s}| \leq \frac{x_{0}^{2}}{2}\left(\frac{1}{t_{0}-s}-\frac{1}{t_{0}-\tilde{s}}\right) \Longleftrightarrow \frac{L^{2}}{D^{2}} \leq 1-\varepsilon,
$$

which is satisfied for small enough $\varepsilon$ since (32) amounts to $|D|>L$.

\section{References}

[1] A. D. Alexandrov. Almost everywhere existence of the second differential of a convex function and some properties of convex surfaces connected with it. Leningrad State Univ. Annals [Uchenye Zapiski] Math. Ser., 6:3-35, 1939.

[2] L. Ambrosio, C. De Lellis, and C. Mantegazza. Line energies for gradient vector fields in the plane. Calc. Var. Partial Differential Equations, 9(4):327$355,1999$. 
[3] L. Ambrosio, N. Fusco, and D. Pallara. Functions of bounded variation and free discontinuity problems. Oxford Mathematical Monographs. The Clarendon Press, Oxford University Press, New York, 2000.

[4] L. Ambrosio, B. Kirchheim, M. Lecumberry, and T. Rivière. On the rectifiability of defect measures arising in a micromagnetics model. In Nonlinear problems in mathematical physics and related topics, II, volume 2 of Int. Math. Ser. (N. Y.), pages 29-60. Kluwer/Plenum, New York, 2002.

[5] G. Bellettini, L. Bertini, M. Mariani, and M. Novaga. Г-entropy cost for scalar conservation laws. Arch. Ration. Mech. Anal., 195(1):261-309, 2010.

[6] S. Conti and C. De Lellis. Sharp upper bounds for a variational problem with singular perturbation. Math. Ann., 338(1):119-146, 2007.

[7] M.G. Crandall, H. Ishii, and P.-L. Lions. User's guide to viscosity solutions of second order partial differential equations. Bull. Amer. Math. Soc. (N.S.), 27(1):1-67, 1992.

[8] M.G. Crandall and P.-L. Lions. Viscosity solutions of Hamilton-Jacobi equations. Trans. Amer. Math. Soc., 277(1):1-42, 1983.

[9] C. De Lellis and R. Ignat. A regularizing property of the $2 D$-eikonal equation. Comm. Partial Differential Equations, 40(8):1543-1557, 2015.

[10] C. De Lellis and F. Otto. Structure of entropy solutions to the eikonal equation. J. Eur. Math. Soc., 5(2):107-145, 2003.

[11] C. De Lellis, F. Otto, and M. Westdickenberg. Structure of entropy solutions for multi-dimensional scalar conservation laws. Arch. Ration. Mech. Anal., 170(2):137-184, 2003.

[12] C. De Lellis, F. Otto, and M. Westdickenberg. Minimal entropy conditions for Burgers equation. Quart. Appl. Math., 62(4):687-700, 2004.

[13] C. De Lellis and T. Rivière. The rectifiability of entropy measures in one space dimension. J. Math. Pures Appl. (9), 82(10):1343-1367, 2003.

[14] C. De Lellis and M. Westdickenberg. On the optimality of velocity averaging lemmas. Ann. Inst. H. Poincaré Anal. Non Linéaire, 20(6):1075-1085, 2003.

[15] A. DeSimone, S. Müller, R. Kohn, and F. Otto. A compactness result in the gradient theory of phase transitions. P. Roy. Soc. Edinb. A, 131(04):833-844, 2001.

[16] L. Giacomelli and F. Otto. New bounds for the Kuramoto-Sivashinsky equation. Comm. Pure Appl. Math., 58(3):297-318, 2005.

[17] F. Golse and B. Perthame. Optimal regularizing effect for scalar conservation laws. Rev. Mat. Iberoam., 29(4):1477-1504, 2013.

[18] R. Ignat and B. Merlet. Entropy method for line-energies. Calc. Var. Partial Differential Equations, 44(3-4):375-418, 2012.

[19] P.-E. Jabin, F. Otto, and B. Perthame. Line-energy Ginzburg-Landau models: zero-energy states. Ann. Sc. Norm. Super. Pisa Cl. Sci. (5), 1(1):187$202,2002$. 
[20] P.-E. Jabin and B. Perthame. Compactness in Ginzburg-Landau energy by kinetic averaging. Comm. Pure Appl. Math., 54(9):1096-1109, 2001.

[21] P.-E. Jabin and B. Perthame. Regularity in kinetic formulations via averaging lemmas. ESAIM Control Optim. Calc. Var., 8:761-774, 2002. A tribute to J. L. Lions.

[22] W. Jin and R. V. Kohn. Singular perturbation and the energy of folds. J. Nonlinear Sci., 10(3):355-390, 2000.

[23] S. N. Kružkov. First order quasilinear equations with several independent variables. Mat. Sb. (N.S.), 81 (123):228-255, 1970.

[24] M. Lecumberry. Geometric structure of micromagnetic walls and shock waves in scalar conservation laws. PhD thesis, Université de Nantes, 2004.

[25] P.-L. Lions. Generalized solutions of Hamilton-Jacobi equations, volume 69 of Research Notes in Mathematics. Pitman (Advanced Publishing Program), Boston, Mass.-London, 1982.

[26] P.-L. Lions, B. Perthame, and E. Tadmor. A kinetic formulation of multidimensional scalar conservation laws and related equations. J. Amer. Math. Soc., 7(1):169-191, 1994.

[27] M. Mariani. Large deviations principles for stochastic scalar conservation laws. Probab. Theory Related Fields, 147(3-4):607-648, 2010.

[28] C.P. Niculescu and L.-E. Persson. Convex functions and their applications. CMS Books in Mathematics/Ouvrages de Mathématiques de la SMC, 23. Springer, New York, 2006.

[29] O. A. Oleĭnik. Discontinuous solutions of non-linear differential equations. Uspehi Mat. Nauk (N.S.), 12(3(75)):3-73, 1957.

[30] A. Poliakovsky. Upper bounds for a class of energies containing a non-local term. ESAIM Control Optim. Calc. Var., 16(4):856-886, 2010.

[31] T. Rivière. Parois et vortex en micromagnétisme. Exp. No. XIV, 15. In Journées "Équations aux Dérivées Partielles" (Forges-les-Eaux, 2002). Univ. Nantes, Nantes, 2002.

[32] L Tartar. The compensated compactness method applied to systems of conservation laws. In Systems of nonlinear partial differential equations (Oxford, 1982), volume 111 of NATO Advanced Science Institutes Series C: Mathematical and Physical Sciences, pages 263-285, 1983.

[33] S.R.S. Varadhan. Large deviations for the asymmetric simple exclusion process. In Stochastic analysis on large scale interacting systems, volume 39 of Adv. Stud. Pure Math., pages 1-27. Math. Soc. Japan, Tokyo, 2004. 